Diez, P., Parés, N. and Huerta, A., Error Estimation and Quality Control, Encyclopedia of Aerospace Engineering, Wiley, Vol. 3, Part 15, Chapter 144, pp. 1725-1734, 2010

\title{
Error Estimation and Quality Control
}

\author{
P. Díez, N. Parés, A. Huerta \\ Universitat Politècnica de Catalunya, Laboratori de Càlcul Numèric \\ Departament de Matemàtica Aplicada III, E.T.S.E. de Camins, Canals i Ports \\ Campus Nord, Jordi Girona 1, 08034 Barcelona, Spain
}

\begin{abstract}
This chapter provides a description of the error assessment tools available for general Finite Element Analysis, in particular those for solid and structural mechanics. The text focuses in goal-oriented error estimation, in terms of general quantities of interest rather that in energy norm. However, the energy norm estimates are also discussed because they are seen as basic tools that must be used for assessing the error in arbitrary functional outputs of the solution. Attention is paid to the classification of the different methodologies and their main characteristics.
\end{abstract}

KEY WORDS: Error estimation, quality control, verification and validation, energy norm, goal-oriented adaptivity

\section{INTRODUCTION}

The Verification and Validation keywords pertain to the quality analysis of the numerical solution provided by the Finite Element Method (FEM). Validation refers to the discrepancy between the model (physical/mathematical) and reality (or experiments). This chapter is concentrated in the Verification concept, which concerns the errors introduced by the numerical solver. More precisely, the methodologies assessing the error associated with the finite element discretization are briefly reviewed.

Thus, it is assumed in the following that the error is the difference between the exact and the numerical solutions of some mathematical problem (typically a boundary value problem, that is, a partial differential equation plus some properly posed boundary conditions). Among the different error sources, the discretization errors are the main focus in this chapter, but the solution is also affected by different mistakes and blunders. The latter may come both from the programmer of the code (they are then referred as code bugs) or the user, who sometimes misunderstands the data set to be entered into the code or misinterprets the results furnished by the code. It is beyond of the scope of this chapter analyzing these error sources.

The error introduced by the numerical discretization, the finite element mesh, is assessed using either a priori and a posteriori error estimates. A priori estimates are mathematical expressions relating some measure of the error with the parameters of the discretization, namely, the characteristic element size, $h$, and the degree of the polynomial approximation inside the elements, $p$. Unknown constants are involved in the expressions, independent of $h$ 
and $p$ but depending on the exact solution. A priori estimates are essential tools to analyze the FEM, in particular its convergence behavior. Nevertheless, a priori estimates are not providing information of the actual error for a concrete solution corresponding to a given mesh. This chapter is concentrateed in a posteriori error assessment. These tools require using the approximate solution (they must be used after the finite element computation is performed, a posteriori) and provide information on the actual error associated with this approximation.

The first attempts in a posteriori error assessment did provide approximations of the error measured in energy norm. In the last decade, a huge effort has been produced in assessing the error in arbitrary quantities of interest. This research is extremely useful since it relates with goal-oriented error adaptivity. That is, finding the optimal mesh producing the result specified by the user with the prescribed accuracy at a minimum cost. Moreover, a recently open line of research concentrates in providing certificates of the approximate solution or, conversely, guaranteed bounds in which the exact solution lies.

The remainder of the chapter is devoted to describe the main goals and endeavors of the error assessment techniques, to schematically classify the principal estimators and to review their characteristics and potential.

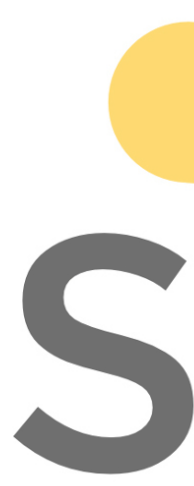

\section{PROBLEM STATEMENT}

The different approaches to error estimation for the finite element numerical approximations
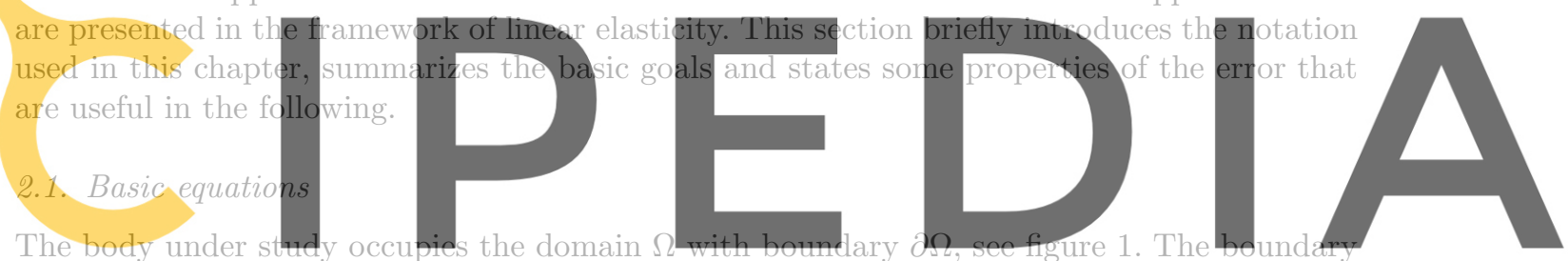

$\partial \Omega$ is divided in two disjoint parts, $\Gamma_{N}$ and $\Gamma_{D}$. In the Dirichlet part of the boundary, $\Gamma_{D}$,

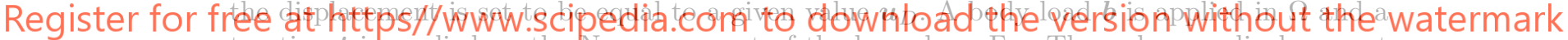

traction $t$ is applied on the Neumann part of the boundary, $\Gamma_{N}$. The unknown displacement

field $u$ and the corresponding stresses $\sigma(u)$ are found by solving the following boundary value problem:

$$
\begin{aligned}
-\boldsymbol{\nabla} \cdot \boldsymbol{\sigma}(\boldsymbol{u}) & =\boldsymbol{b} & & \text { in } \Omega, \\
\boldsymbol{\sigma}(\boldsymbol{u}) \cdot \boldsymbol{n} & =\boldsymbol{t} & & \text { on } \Gamma_{N}, \\
\boldsymbol{u} & =\boldsymbol{u}_{D} & & \text { on } \Gamma_{D} .
\end{aligned}
$$

The variational or weak form of problem (1) requires introducing the following functional spaces. The space of admissible displacements $\mathcal{U}$ (a subspace of $\mathcal{H}^{1}(\Omega)$ of functions fulfilling (1c)) and the space of virtual displacements, $\mathcal{V}$ (also known as trial functions, similar to $\mathcal{U}$ but vanishing on $\Gamma_{D}$ ). Thus the weak form is readily expressed as find $\boldsymbol{u} \in \mathcal{U}$ such that

$$
a(\boldsymbol{u}, \boldsymbol{v})=l(\boldsymbol{v}), \text { for all } \boldsymbol{v} \in \mathcal{V},
$$

where

$$
a(\boldsymbol{u}, \boldsymbol{v}):=\int_{\Omega} \boldsymbol{\sigma}(\boldsymbol{u}): \boldsymbol{\epsilon}(\boldsymbol{v}) d \Omega \quad, \quad l(\boldsymbol{v}):=\int_{\Omega} \boldsymbol{b} \cdot \boldsymbol{v} d \Omega+\int_{\Gamma_{N}} \boldsymbol{t} \cdot \boldsymbol{v} d \Gamma
$$

Encyclopedia of Aerospace Engineering. Edited by Richard Blockley and Wei Shyy.

(C) 2010 John Wiley \& Sons, Ltd. 


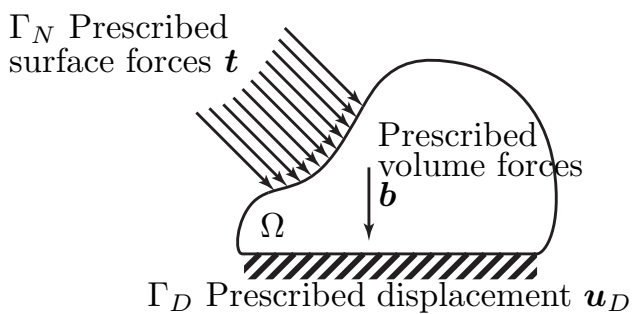

Figure 1. Representation of the structure

being $\boldsymbol{\epsilon}(\cdot)$ the strain operator. Recall that the Hooke tensor $\mathbb{C}$ relates stresses and strains,

$$
\boldsymbol{\sigma}(\boldsymbol{u})=\mathbb{C}: \epsilon(\boldsymbol{u})
$$

It is useful expressing the bilinear form $a\left(\cdot,{ }^{\circ}\right)$ in terms of stresses instead of displacements by formally introducing $\bar{a}(\cdot, \cdot)$ such that

$$
\bar{a}(\sigma, \tau):=\int_{\Omega} \sigma: \mathbb{C}^{-1}: \tau d \Omega .
$$

Note that, with this definition, $a(\boldsymbol{u}, \boldsymbol{v})=\bar{a}(\boldsymbol{\sigma}(\boldsymbol{u}), \boldsymbol{\sigma}(\boldsymbol{v}))$.

A finite element mesh of characteristic size $h$ discretizing $\Omega$ induces the functional spaces
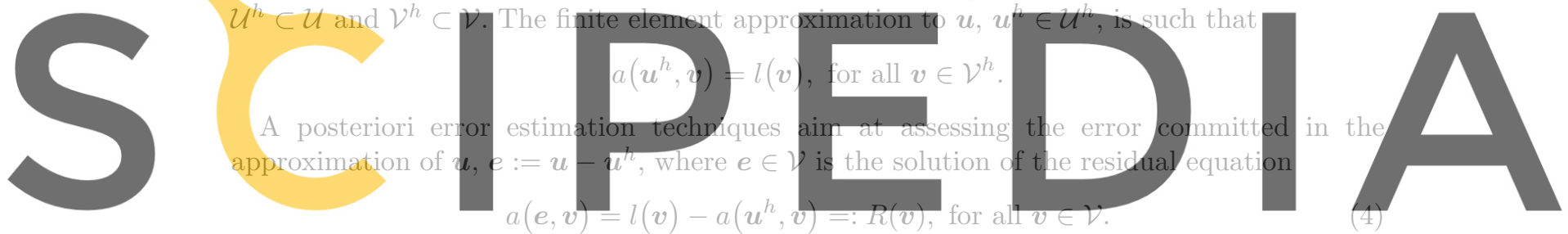

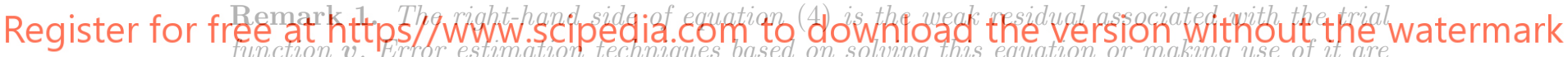

hence named residual type error estimators. It is worth noting also that the weak residual is also

expressed in terms of the elementary strong residuai $\boldsymbol{r}_{e l}=\boldsymbol{b}+\nabla \cdot \boldsymbol{\sigma}\left(\boldsymbol{u}^{h}\right)$ ( which can be evaluated in the interior of the elements $\Omega_{k}, k=1,2, \ldots, n_{e l}$, of the mesh) and the singular residual, $\boldsymbol{r}_{\text {sing. }}$. The singular residual is defined as the jump of the normal component of $\boldsymbol{\sigma}\left(\boldsymbol{u}^{h}\right)$ on the interelement edges $\gamma$ (sides in 3D) in $\Gamma_{\text {int }}, \boldsymbol{r}_{\text {sing }}=\llbracket \boldsymbol{\sigma}\left(\boldsymbol{u}^{h}\right) \cdot \boldsymbol{n} \rrbracket_{\Gamma_{\text {int }}}$ and as the non verification of the Neumann boundary condition on the element edges $\gamma$ in $\Gamma_{N}, \boldsymbol{r}_{\text {sing }}=\boldsymbol{t}-\boldsymbol{\sigma}\left(\boldsymbol{u}^{h}\right) \cdot \boldsymbol{n}$. The resulting expression is

$$
R(\boldsymbol{v})=\sum_{k=1}^{n_{e l}} \int_{\Omega_{k}} \boldsymbol{r}_{e l} \cdot \boldsymbol{v} d \Omega+\sum_{\gamma \in \Gamma_{\text {int }} \bigcup \Gamma_{N}} \int_{\gamma} \boldsymbol{r}_{\text {sing }} \cdot \boldsymbol{v} d \Gamma .
$$

These two components of the residual reveal the existence of two different error sources, the elementary and singular residuals. The former is associated with the lack of verification of the differential equation in the interior of the elements, the latter with the non verification of the continuity requirements of the stress field. The main rationale of the explicit residual error estimates consists in evaluating this two terms separately.

Encyclopedia of Aerospace Engineering. Edited by Richard Blockley and Wei Shyy.

(C) 2010 John Wiley \& Sons, Ltd. 
The energy norm of the error, $\|\boldsymbol{e}\|$, is taken as a global measure of the error. This is the norm induced by $a(\cdot, \cdot)$ or $\bar{a}(\cdot, \cdot)$ when applied to stresses, namely

$$
\|e\|^{2}=a(\boldsymbol{e}, \boldsymbol{e})=\bar{a}\left(\boldsymbol{\sigma}_{e}, \boldsymbol{\sigma}_{e}\right)=\|\| \boldsymbol{\sigma}_{e} \|\left.\right|^{2},
$$

where $\boldsymbol{\sigma}_{e}$ is the error in stresses $\boldsymbol{\sigma}_{e}:=\boldsymbol{\sigma}(\boldsymbol{u})-\boldsymbol{\sigma}\left(\boldsymbol{u}^{h}\right)$.

\subsection{Assessing the energy norm of the error}

A first step in a posteriori assessment is estimating the error measured in the energy norm, that is obtaining a good approximation of $\boldsymbol{\sigma}_{e}$ and computing $\|\boldsymbol{e}\|$. This translates in finding a new stress field $\boldsymbol{\sigma}^{\star}$ based on the information at hand, that is $\boldsymbol{\sigma}\left(\boldsymbol{u}^{h}\right)$, and such that $\boldsymbol{\sigma}^{\star}$ approximates the actual stresses $\boldsymbol{\sigma}(\boldsymbol{u})$ much better than $\boldsymbol{\sigma}\left(\boldsymbol{u}^{h}\right)$. Thus, a computable error estimate is readily obtained

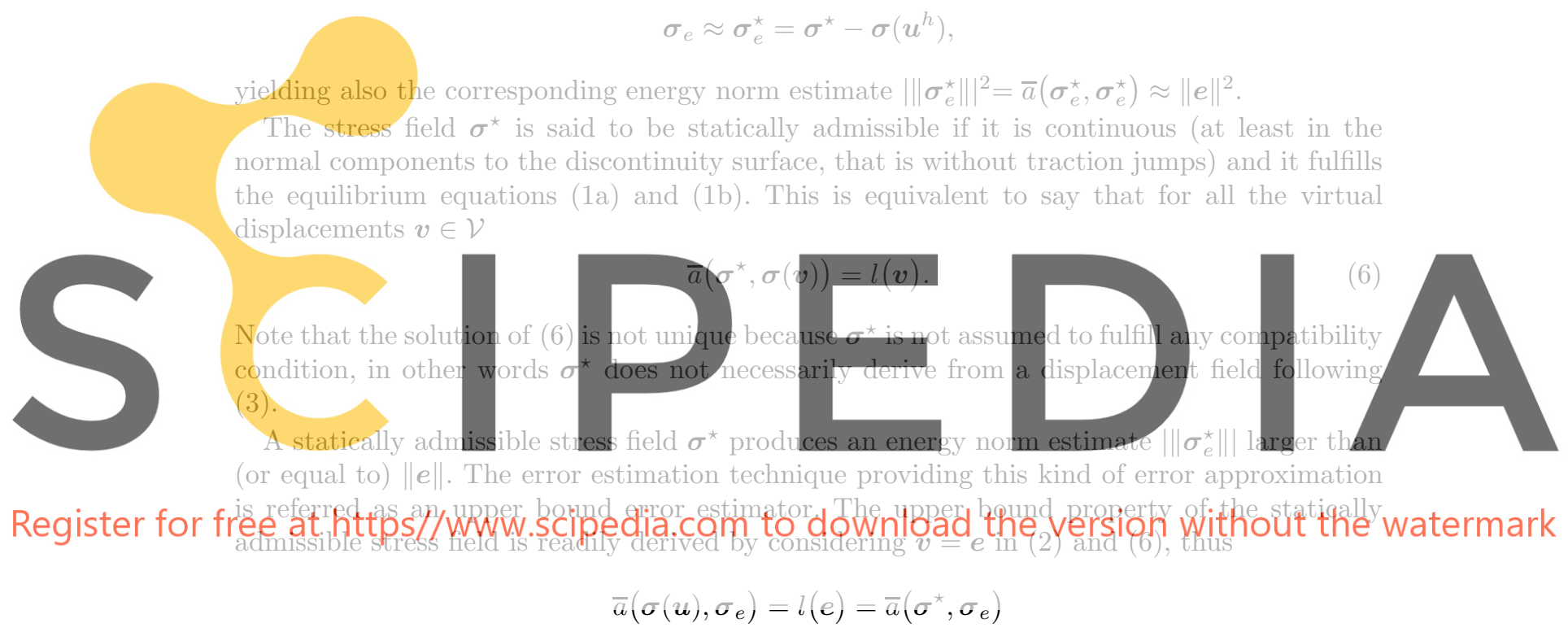

and subtracting $\bar{a}\left(\boldsymbol{\sigma}\left(\boldsymbol{u}^{h}\right), \boldsymbol{\sigma}_{e}\right)$ in both sides

$$
\bar{a}\left(\boldsymbol{\sigma}_{e}, \boldsymbol{\sigma}_{e}\right)=\bar{a}\left(\boldsymbol{\sigma}_{e}^{\star}, \boldsymbol{\sigma}_{e}\right)
$$

which yields $\left|\left\|\boldsymbol{\sigma}_{e}\right\|\right| \leq\left|\left\|\boldsymbol{\sigma}_{e}^{\star}\right\|\right|$ by simply considering the Cauchy-Schwarz inequality.

Thus, the key issue in any error estimation technique is to produce a properly enhanced stress field $\boldsymbol{\sigma}^{\star}$. Moreover, if $\boldsymbol{\sigma}^{\star}$ is build up such that it is statically admissible, then this additional feature confers to the estimator the upper bound property. The strategies producing the enhanced stresses $\boldsymbol{\sigma}^{\star}$ are classified into two categories: recovery type estimators and implicit residual type estimators, which are discussed in sections 3 and 4 .

It is worth remarking that, in general, the enhanced stress $\boldsymbol{\sigma}^{\star}$ and the corresponding stress error $\boldsymbol{\sigma}_{e}^{*}$ can only be used to evaluate the energy norm of the error, and no other quantities. In particular, any magnitude based on the displacement error cannot be evaluated using $\boldsymbol{\sigma}^{\star}$.

Encyclopedia of Aerospace Engineering. Edited by Richard Blockley and Wei Shyy.

(c) 2010 John Wiley \& Sons, Ltd. 


\subsection{Quantities of interest, adjoint problem and error representation}

Assessing the energy norm of the error is not sufficient for many applications. In practice, the finite element user is interested in specific magnitudes extracted from the global solution by some post-process. These magnitudes are referred as quantities of interest or functional outputs. Goal-oriented error assessment strategies aim at estimating the error committed in these quantities and possibly providing bounds for it.

The quantities of interest considered here are linear functional outputs of the solution, $l^{\mathcal{O}}(\boldsymbol{u})$. In particular, those expressed in the form

$$
l^{\mathcal{O}}(\boldsymbol{u})=\int_{\Omega} \boldsymbol{b}^{\mathcal{O}} \cdot \boldsymbol{u} d \Omega+\int_{\Gamma_{N}} \boldsymbol{t}^{\mathcal{O}} \cdot \boldsymbol{u} d \Gamma+a\left(\boldsymbol{u}, \chi^{\mathcal{O}}\right),
$$

where $\boldsymbol{b}^{\mathcal{O}}, \boldsymbol{t}^{\mathcal{O}}$ and $\chi^{\mathcal{O}}$ are given functions characterizing the quantity of interest. Note that $l^{\mathcal{O}}(\cdot)$ has the same structure as the right-hand side of $(2)$. The extension to nonlinear outputs is discussed in Xuan et al., 2006.

This expression is pretty general and accounts for a large variety of quantities of interest. The first term in (7) is a weighted average of the displacements, being $b^{\mathcal{O}}$ the weight. Note that this average is restricted to the support of $b^{\mathcal{O}}$ which is in practice the way of indicating the zone of interest. Similarly, the second term in (7) accounts for averaged displacements along a part of the Neumann boundary. Note that displacements on the Dirichlet boundary, $\Gamma_{D}$, are known a priori and therefore it makes not sense to include in the quantity of interest
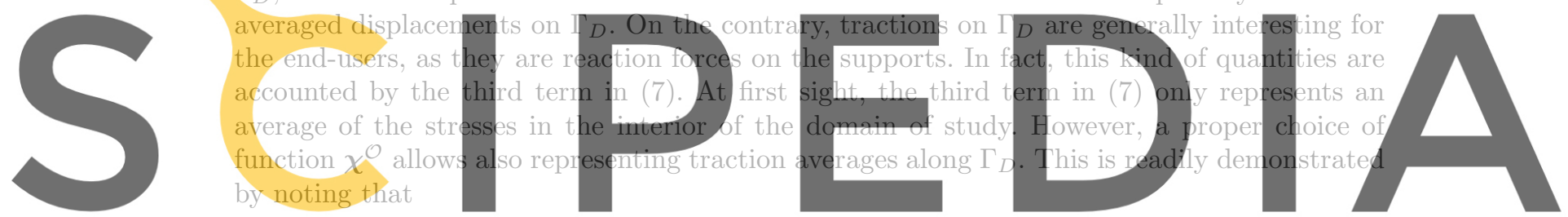

Register for free at https/h/DWww.scipedia.com to downiload the version without the watermark

Equation (8) is obtained after the usual algebraic manipulation, using the weighted residuals technique into the original equation (1), taking $\chi^{\mathcal{O}}$, which does not vanish on $\Gamma_{D}$, as weighting function. It is clear from (8) that the third term in (7) is a traction average on $\Gamma_{D}$ plus a computable term involving part of the data.

The expression (7) allows also determining pointwise quantities by using functions of the Dirac delta type although in practice smeared versions are preferred (averages in neighborhoods of the point) in order to avoid singularities.

The objective of the goal-oriented error assessment is to estimate the value of $l^{\mathcal{O}}(\boldsymbol{e})$ which, for linear outputs, coincides with $l^{\mathcal{O}}(\boldsymbol{u})-l^{\mathcal{O}}\left(\boldsymbol{u}_{H}\right)$.

As pointed out in the previous section, the enhanced stresses $\boldsymbol{\sigma}^{\star}$ can only be used to assess the energy norm of the error. Thus, an error representation is needed to express the error in the quantity of interest in terms of the energy error. This error representation requires introducing an auxiliary problem, denoted as adjoint or dual problem by different authors . This problem reads: find $\psi \in \mathcal{V}$ such that

$$
a(\boldsymbol{v}, \psi)=l^{\mathcal{O}}(\boldsymbol{v}), \text { for all } \boldsymbol{v} \in \mathcal{V} \text {. }
$$

Encyclopedia of Aerospace Engineering. Edited by Richard Blockley and Wei Shyy.

(C) 2010 John Wiley \& Sons, Ltd. 
Note that the adjoint solution $\boldsymbol{\psi}$ lies in the space $\mathcal{V}$ (that is vanishes on $\Gamma_{D}$ ) and that, for the sake of clarity, the order of the arguments in $a(\cdot, \cdot)$ is switched with respect to the original equation (2). The numerical solution of the adjoint problem (9), $\boldsymbol{\psi}^{h}$, has the associated error $\varepsilon:=\boldsymbol{\psi}-\boldsymbol{\psi}^{h}$. These auxiliary functions are introduced such that the following error representation holds:

$$
l^{\mathcal{O}}(\boldsymbol{e})=a(\boldsymbol{e}, \boldsymbol{\psi})=a(\boldsymbol{e}, \boldsymbol{\varepsilon}) .
$$

This error representation allows bounding the error in terms of the energy norm of the errors in the direct and adjoint problem. This is a direct consequence of the Cauchy-Schwarz inequality, namely

$$
\left|l^{\mathcal{O}}(\boldsymbol{e})\right|=|a(\boldsymbol{e}, \boldsymbol{\varepsilon})| \leq\|\boldsymbol{e}\|\|\varepsilon\| .
$$

An obvious error bound for the quantity of interest follows: $l^{\mathcal{O}}(\boldsymbol{e})$ ranges between $\pm\|\boldsymbol{e}\|\|\varepsilon\|$. Thus, an upper bound of the quantity of interest (in absolute value) is obtained if upper bounds for $\|e\|$ and $\|\varepsilon\|$ are available. The sharpness of this upper and lower bounding of the error in the quantity of interest is improved by considering the so-called parallelogram identity:

$$
l^{\mathcal{O}}(e)=\frac{1}{4}\left\|\kappa e+\frac{1}{\kappa} \varepsilon\right\|^{2}-\frac{1}{4}\left\|\kappa e-\frac{1}{\kappa} \varepsilon\right\|^{2}
$$

standing for any non-zero factor $\kappa$. It follows from (11) that an upper bound for $l^{\mathcal{O}}(e)$ is obtained by combining an upper bound for $\left\|\kappa e+\frac{1}{\kappa} \varepsilon\right\|$ and a lower bound for $\left\|\kappa e-\frac{1}{\kappa} \varepsilon\right\|$ (using
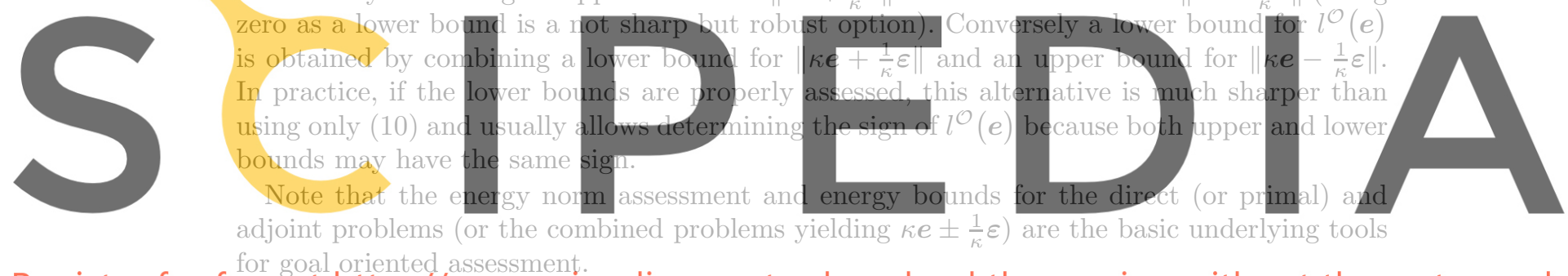

\section{RECOVERY ESTIMATES}

The so-called recovery or flux projection error estimates use a simple postprocess technique to recover an enhanced stress field, $\boldsymbol{\sigma}^{\star}$, as introduced in 2.2. Using the pioneering idea introduced in Zienkiewicz and Zhu, 1987, $\boldsymbol{\sigma}^{\star}$ is straightforwardly computed as a least squares fitting of $\boldsymbol{\sigma}\left(\boldsymbol{u}^{h}\right)$.

Note that the computed stresses $\boldsymbol{\sigma}\left(\boldsymbol{u}^{h}\right)$ are discontinuous across the interelement edges or sides. The corresponding traction jumps are in fact the basis for the singular residual, $\boldsymbol{r}_{\text {sing, }}$, as shown in (5), and one of the error sources. Note also that the stresses inside the elements are computed from the derivatives of the displacements and therefore they are of lower polynomial degree: for linear elements stresses are piecewise constant, for quadratic elements stresses are linear... This is related with the interior residual $\boldsymbol{r}_{\mathrm{el}}$ : for instance, for linear elements $\boldsymbol{r}_{\mathrm{el}}=\boldsymbol{b}$. It is clear that in order to enhance the stresses one has to smooth out the discontinuities (suppress traction jumps) and to increase the polynomial degree of the stress approximation inside the elements.

Encyclopedia of Aerospace Engineering. Edited by Richard Blockley and Wei Shyy. (C) 2010 John Wiley \& Sons, Ltd. 
This objective is easily reached by describing the stresses with the same functional description used for the displacements. A discrete space for the stresses $\mathcal{S}^{h}$ is introduced such that every component of the stress field is described using the same interpolation as the components of $\mathcal{U}^{h}$ : functions in $\mathcal{S}^{h}$ are of the same type as functions in $\mathcal{U}^{h}$ (continuous and piecewise polynomial).

Thus, the recovered stress $\boldsymbol{\sigma}^{\star}$ is selected in $\mathcal{S}^{h}$ such that it minimizes the error $\boldsymbol{\sigma}^{\star}-\boldsymbol{\sigma}\left(\boldsymbol{u}^{h}\right)$ in a least squares sense. Following this basic idea, different fitting criteria have been introduced by different authors. Among the more popular it is worth mentioning the SPR (Super Patch Recovery) introduced in Zienkiewicz and Zhu, 1987. The SPR is based on the local polynomial fitting of the stress field in patches of elements. The locally fitted stress is evaluated at the nodal points to determine the nodal values of $\sigma^{\star} \in \mathcal{S}^{h}$.

A different approach to recovery estimates was introduced in Wiberg et al., 1996 for structural dynamics. In this context the stress recovery does not suffice to compute the error; the error in displacements and velocities is also required. Thus, a technique enhancing the displacement approximation provided by the FEM is introduced. This technique follows the same rationale: the displacements $\boldsymbol{u}^{h}$ are less regular than expected (mathematically speaking they are $\mathcal{C}^{0}$ and not $\mathcal{C}^{1}$ ) and their local polynomial degree is eventually too low. The enhanced displacements $u^{\star}$ are build-up as a post-process of $\boldsymbol{u}^{h}$ increasing the regularity requirements and the enriching the degree of the local polynomial description. This is essentially performed at every element of the mesh assessing the local curvatures of the solution, fitting a least squares polynomial in a patch of elements centered in the element under study. Recently, this approach
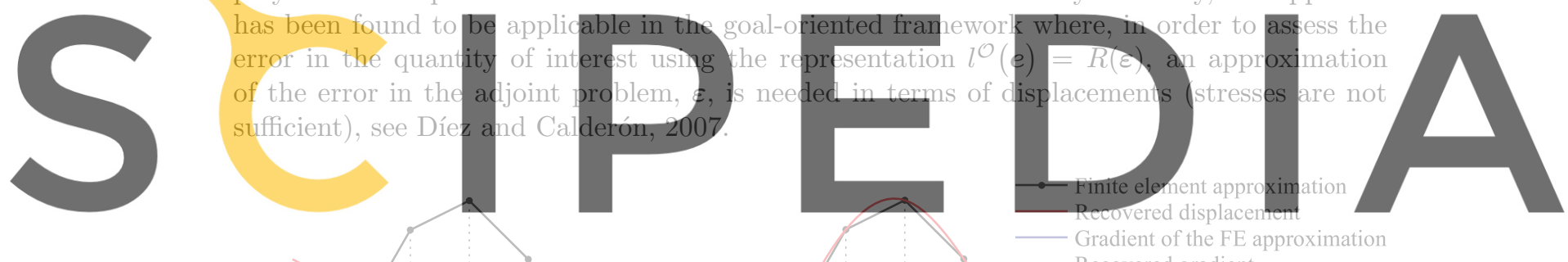

Register for free àt httpș//www:scipedia.com to download the version without the watermark

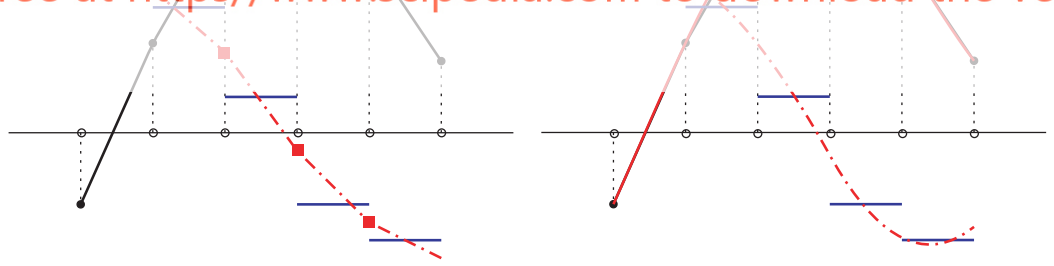

Figure 2. Illustration of the flux projection (left) and enhancement of displacement (right) recovery estimates

\section{RESIDUAL TYPE ESTIMATES}

As suggested by their name, residual type estimators assess the error using the residual, either using expression (5) (explicit residual estimates) or solving approximately (4) (implicit residual estimates).

Encyclopedia of Aerospace Engineering. Edited by Richard Blockley and Wei Shyy. (c) 2010 John Wiley \& Sons, Ltd. 


\subsection{Explicit estimates}

Explicit estimates are based on the decomposition of the weak residual discussed in remark 1. The computable elementary residual $\boldsymbol{r}_{\mathrm{el}}$ and singular residual $\boldsymbol{r}_{\text {sing }}$ are seen as the two sources of error. Explicit estimates are based on postprocessing these two quantities and getting an approximation to the error. Thus, the estimate does not require solving any local problem and is computed directly from the finite element approximation. The input data of the problem to be solved is required to compute the elementary residual, and the part of the singular residual associated with the Neumann boundary. Note that this information is not used in the recovery estimates discussed in section 3, which are computed using only $\boldsymbol{\sigma}\left(\boldsymbol{u}^{h}\right)$ and no use is made of the data of the original problem.

The idea of explicit residual estimates uses (5) for $v=e-\Pi^{h} e\left(\Pi^{h}\right.$ stands for the interpolation operator in $\mathcal{V}^{h}$ ) together with the Cauchy-Schwarz inequality and the a priori interpolation estimates. Cooking all these ingredients, the following expression is found (see Ainsworth and Oden, 2000 for a detailed derivation)

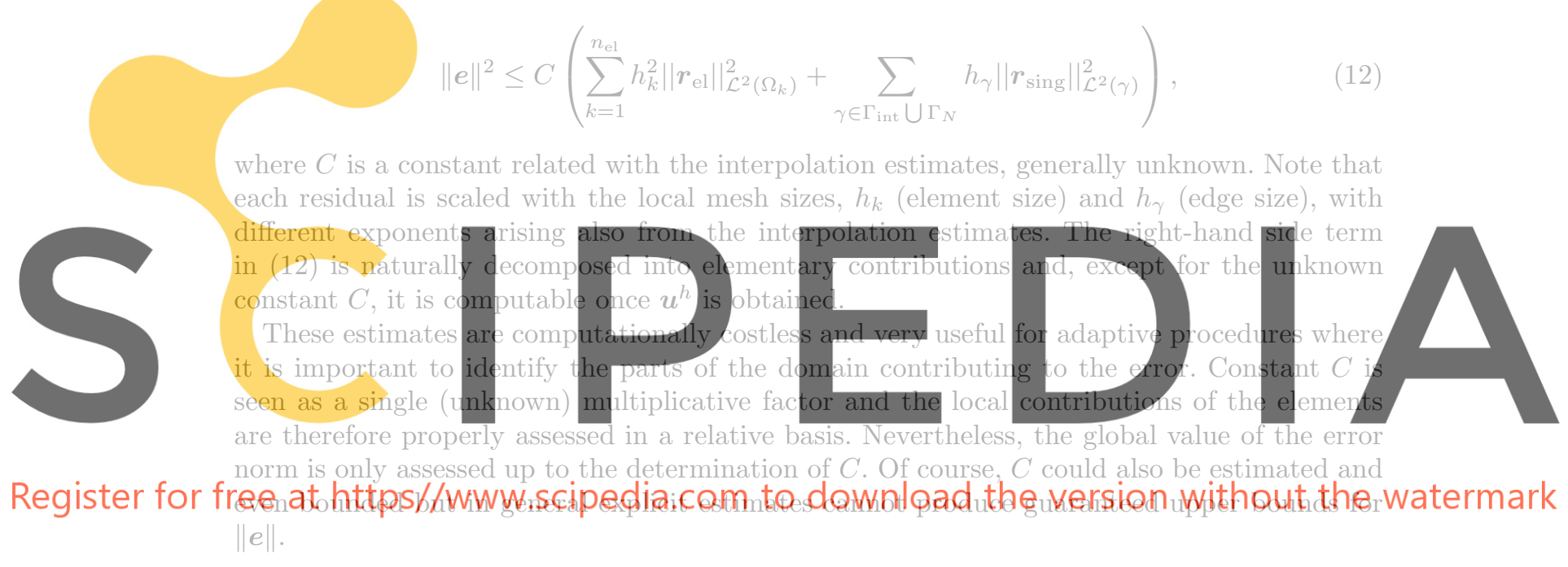

\subsection{Implicit estimates}

Implicit estimators aim at avoiding the disadvantages of explicit estimates by solving the original error equation (4) in a local basis. That is, typically in small domains (the elements or patches of elements) in which a local version of (4) is solved numerically. This requires locally increasing the resolution with respect to the original approximation in $\mathcal{U}^{h}$. The implicit estimates are classified in different categories, depending on

- the domain in which the local problem is stated: element residual methods (solved element by element) and subdomain residual methods (solved in patches of elements, either centered in nodes or elements)

- the boundary conditions imposed on the local problems: either Dirichlet or Neumann. Roughly speaking, the Dirichlet methods provide continuous approximations to the displacement error and lower bounds of the energy and the Neumann methods yield statically admissible stress fields and upper bounds of the energy error

Encyclopedia of Aerospace Engineering. Edited by Richard Blockley and Wei Shyy.

(C) 2010 John Wiley \& Sons, Ltd. 
- the numerical method used to approximate the solution of the local problem: either a standard FE method providing a displacement based approximation of the error (producing the so-called asymptotic estimates which have bounding properties only with respect to a reference solution, not with respect to the exact error) or a dual approach yielding an approximation of the stress field exactly fulfilling the equilibrium equations (producing guaranteed or strict error bounds).

It is worth mentioning here the pioneering work of Ladevèze introducing the error estimators based in the concept of constitutive relation error, see Ladevèze and Leguillon, 1983. This family of error estimators is classified here in the implicit residual framework, together with the estimators solving elementary problems with Neumann boundary conditions, because it perfectly matches the category. The rationale for the presentation and the derivation of these techniques is however pretty different. Following this line of thought, based also in mechanical arguments, strategies to generalize these tools to nonlinear and transient problems have been suggested, see Chamoin and Ladevèze, 2008.

An alternative approach fitting also the implicit residual philosophy are the so-called dual global solvers. This strategy is based on the ideas introduced by Fraeijs de Veubeke, 1965. A statically admissible stress field $\sigma^{\star}$ is obtained by means of a global computation over a discrete space $\mathcal{S}^{h}$ (where the stresses are interpolated). This requires solving a global optimization problem reading: find $\sigma^{\star} \in \mathcal{S}^{h}$ such that the complementary energy $\left|\left\|\sigma^{\star}\right\|\right|^{2}=\bar{a}\left(\sigma^{\star}, \sigma^{\star}\right)$ is minimum, with the additional restriction of being statically admissible, see (6). Thus,
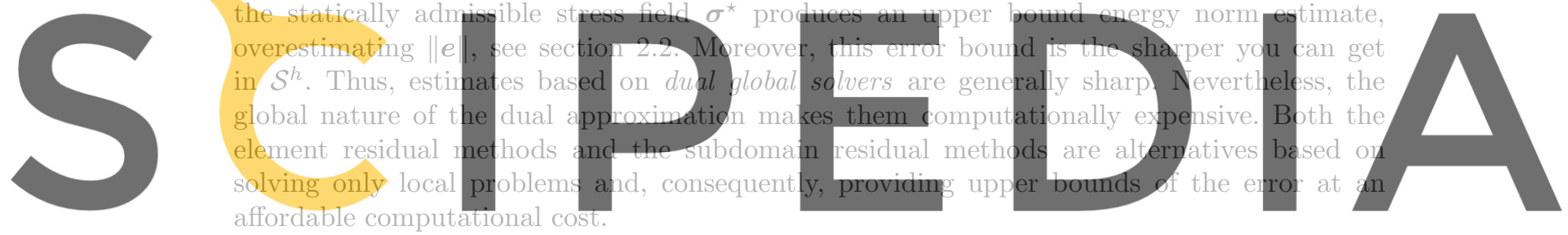

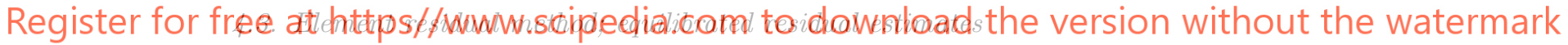

The local version of the error equation (4) in the element $\Omega_{k}$ of the mesh states that the restriction of the error $e$ to $\Omega_{k}$ fulfills

$$
a_{k}(\boldsymbol{e}, \boldsymbol{v})=l_{k}(\boldsymbol{v})-a_{k}\left(\boldsymbol{u}^{h}, \boldsymbol{v}\right)+\int_{\partial \Omega_{k} \backslash \partial \Omega}(\boldsymbol{\sigma}(\boldsymbol{u}) \cdot \boldsymbol{n}) \cdot \boldsymbol{v} d \Gamma
$$

for all $v$ taking values in $\Omega_{k}$. Subscript $k$ in the linear and bilinear forms indicates that the corresponding integrals are restricted to $\Omega_{k}$. Note that the last term of the right-hand side stands for the local Neumann boundary conditions and depends on the unknown traction associated with the exact solution. Note also that the local error stress field $\boldsymbol{\sigma}(\boldsymbol{e})$ fulfills a variant of (13), substituting the left-hand side term by $\bar{a}_{k}(\boldsymbol{\sigma}(\boldsymbol{e}), \boldsymbol{\sigma}(\boldsymbol{v}))$.

In order to obtain a solvable local problem, the unknown boundary traction $\boldsymbol{\sigma}(\boldsymbol{u}) \cdot \boldsymbol{n}$ on the boundary of $\Omega_{k}$ is replaced by some approximated value $\boldsymbol{g}_{k}$ that has to be determined on all the interelement edges. Thus, the local equation for the approximated stress error, $\boldsymbol{\sigma}_{e}^{\star}$ is

$$
\bar{a}_{k}\left(\boldsymbol{\sigma}_{e}^{\star}, \boldsymbol{\sigma}(\boldsymbol{v})\right)=l_{k}(\boldsymbol{v})-a_{k}\left(\boldsymbol{u}^{h}, \boldsymbol{v}\right)+\int_{\partial \Omega_{k} \backslash \partial \Omega} \boldsymbol{g}_{k} \cdot \boldsymbol{v} d \Gamma .
$$

Encyclopedia of Aerospace Engineering. Edited by Richard Blockley and Wei Shyy.

(c) 2010 John Wiley \& Sons, Ltd. 
In order to provide statically admissible stresses, the approximated traction $\boldsymbol{g}_{k}$ has to fulfill two properties

1. on the common edge of two contiguous elements $\Omega_{k}$ and $\Omega_{k^{\prime}}, \boldsymbol{g}_{k}=-\boldsymbol{g}_{k^{\prime}}$ (this is to guarantee the continuity of the traction associated with $\sigma^{\star}$ )

2. the boundary traction must be in equilibrium with the interior loads. This compatibility condition is needed to ensure that the problem (14) is solvable.

The compatibility condition requires $\boldsymbol{g}_{k}$ to fulfill

$$
l_{k}(\boldsymbol{v})-a_{k}\left(\boldsymbol{u}^{h}, \boldsymbol{v}\right)+\int_{\partial \Omega_{k} \backslash \partial \Omega} \boldsymbol{g}_{k} \cdot \boldsymbol{v} d \Gamma=0
$$

for any rigid body motion $\boldsymbol{v}$ (in 2D, this means $\boldsymbol{v}$ taking the values of the two translations $\boldsymbol{t}_{x}$ and $t_{y}$ and the rotation $\theta$ ). If this condition is fulfilled, problem (14) is solvable (the solution exists, even if it is not unique). Any of the solutions of this problem produces an upper bound estimate.

The first idea to determine $g_{k}$ was introduced by Bank and Weiser, 1985 and consists in taking $\boldsymbol{g}_{k}$ equal to the average of the numerical normal traction, computed from $\sigma\left(\boldsymbol{u}^{h}\right)$. This is equivalent to assume that $\sigma(u) \cdot n \approx\left\langle\sigma\left(u^{h}\right)\right\rangle_{\text {ave }} \cdot n$ on the interelement edges. This option fulfills the continuity restriction but fails guaranteing the compatibility condition (15). To overcome this problem, Bank and Weiser, 1985 propose the following work-around: the test function $v$ in problem (14) is taken in a restricted functional space of functions vanishing at the
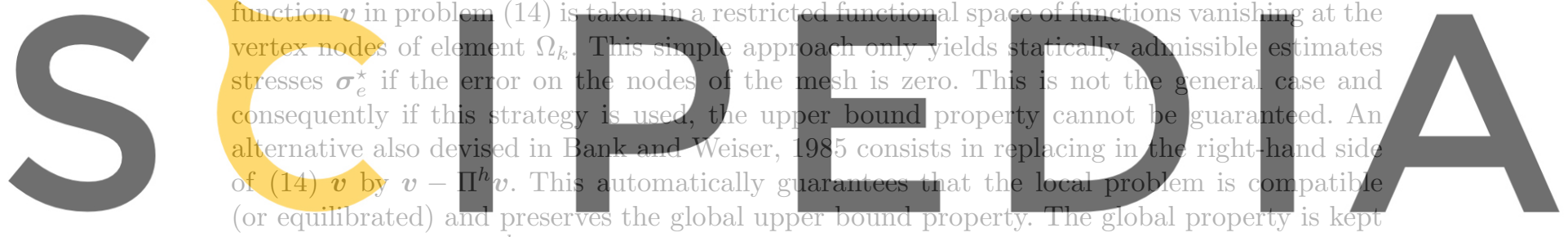

because subtracting $\Pi^{h} v$ in the argument of $R(\cdot)$ (the right-hand side of $(4)$ ) does not chanore.

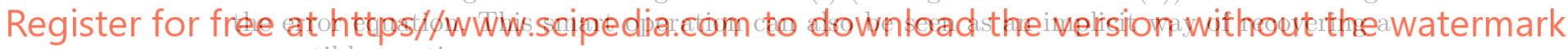
compatible traction $g_{k}$.

This is the basis of the so-called equitibrated residual estimates. In fact, this family of estimators introduces efficient and practical algorithms for constructing equilibrated fluxes, that is recovering $\boldsymbol{g}_{k}$ by solving only local problems. The compatibility condition (15) is at the first sight a global restriction, involving the tractions on all the element boundaries. If the equilibrated residual methods are among the most popular implicit residual type estimators is because the computation of the tractions $\boldsymbol{g}_{k}$ is decoupled node to node. Using a smart representation of $\boldsymbol{g}_{k}$, the nodal contributions to $\boldsymbol{g}_{k}$ on all the edges converging in a given node are computed independently, and it requires solving a small linear system of equations as indicated in Ainsworth and Oden, 1993; Ladevèze and Leguillon, 1983; Ladevèze et al., 1991; Ladevèze and Maunder, 1996; Sauer-Budge et al., 2004; Parés et al., 2006.

\subsection{Subdomain residual methods; flux-free estimates}

The effectivity of the equilibrated residual method depends on the quality of the local tractions $\boldsymbol{g}_{k}$. For instance, the dual-global estimates are usually much sharper than the equilibrated residual estimates. Moreover, although computing $\boldsymbol{g}_{k}$ as indicated above is computationally

Encyclopedia of Aerospace Engineering. Edited by Richard Blockley and Wei Shyy.

(C) 2010 John Wiley \& Sons, Ltd. 
Element residual method

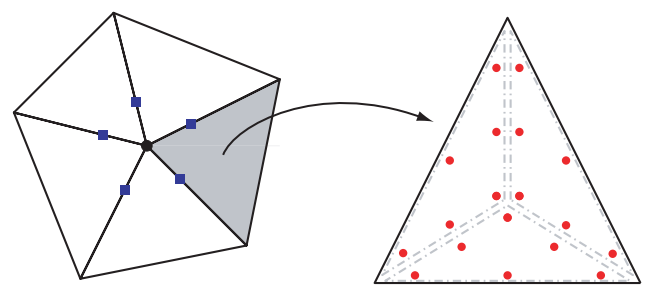

Subdomain residual method

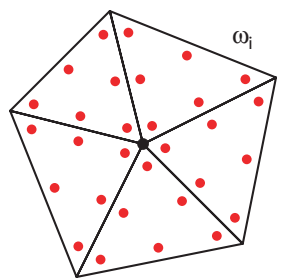

Figure 3. Illustration of the element residual method (left) and the subdomain residual method (right). In the element residual method the contribution to the tractions $\boldsymbol{g}_{k}$ of every node of the mesh (represented by the blue squares) are computed in a nodal basis. Then, the tractions $\boldsymbol{g}_{k}$ are used to solve the local elementary problems and the stresses inside the element fulfilling the equilibrium are determined. Subdomain residual method: a larger local problem is solved for each node of the mesh but no equilibrated tractions have to be computed. The red circles represent the degrees of freedom describing the approximated stresses.

inexpensive because the local problems are decoupled, the implementation of the equilibration techniques is often involved and difficult to generalize to different element types or space dimensions.

The subdomain residual methods are introduced as an alternative to equilibrated residual methods such that:

- they preclude solving a global problem (the local equations are posed in different subdomains, patches of elements surrounding a node, also denoted as stars)

- they provide upper bound estimates

- they circumvent the necessity of finding proper tractions as boundary conditions for the local problems. The local boundary conditions are natural and the estimates are also said to be flux-free.

In order to localize the error equation (4), use is made of the partition of unity property. Let $\phi_{i}$ be the linear finite element interpolation function associated with the $i$-th vertex node of the mesh. Note that these functions sum up to the unity and that the support of $\phi_{i}$ is precisely the patch of elements containing this node, $\omega_{i}$. Thus, a local version of (4) in $\omega_{i}$, providing a local approximation $\boldsymbol{\sigma}_{e}^{\star i}$ of the stress error, is readily recovered as

$$
\bar{a}_{\omega^{i}}\left(\boldsymbol{\sigma}_{e}^{\star i}, \boldsymbol{\sigma}(\boldsymbol{v})\right)=R\left(\phi_{i} \boldsymbol{v}\right)
$$

for all $\boldsymbol{v}$ taking values in $\omega_{i}$, being $\bar{a}_{\omega^{i}}(\cdot, \cdot)$ the restriction of $\bar{a}(\cdot, \cdot)$ to $\omega_{i}$. The sum of the local approximations to the stress error $\boldsymbol{\sigma}_{e}^{\star i}$ provide a statically admissible stress field $\boldsymbol{\sigma}_{e}$ and its corresponding error norm is a sharp upper bound of the error, see Parés et al., 2006. The local problem (16) is automatically equilibrated in most of the cases because the right-hand side vanishes for $\boldsymbol{v}$ equal to a rigid body motion. In the unique case in which this equilibrium is not automatically guaranteed (linear elements for structural mechanics) a straightforward modification is introduced to ensure solvability, see Parés et al., 2006.

Similar approaches are developed taking $\bar{a}_{\omega^{i}}(\cdot, \cdot)$ as a locally weighted version of $\bar{a}(\cdot, \cdot)$, see Carstensen and Funken, 1999; Machiels et al., 2000; Morin et al., 2003. In this case the upper bound estimate is obtained adding the squared norms of the local contributions rather than 
adding the functions and computing the norm afterwards. The estimates obtained following this rationale are not as sharp as the ones obtained taking $\bar{a}_{\omega^{i}}(\cdot, \cdot)$ as simple restriction of $\bar{a}(\cdot, \cdot)$.

\subsection{Lower bounds for the energy using implicit Dirichlet estimates}

Recall that in order to get sharp bounds of the error in the quantities of interest using (11), it is important to obtain lower bounds of the energy norm of the error. Any continuous approximation of the displacement error, $e^{\star} \in \mathcal{V}$, is such that $R\left(e^{\star}\right)\left\|e^{\star}\right\|^{-1} \leq\|\boldsymbol{e}\|$. This is a direct consequence of taking $\boldsymbol{v}=\boldsymbol{e}^{\star}$ in (4) (this is only possible if $\boldsymbol{e}^{\star}$ is continuous) and use the Cauchy-Schwarz inequality. Thus, a lower bound is easily recovered after $e^{\star}$.

The simplest way of guaranteeing continuity by solving local residual problems is to use homogeneous Dirichlet boundary conditions (prescribe displacements equal to zero) on the boundary of the local subdomains. This idea was used in Díez et al., 1998 solving such problems elementwise and then complementing the estimate by adding the contribution of a new family of subdomains overlapping the elements while keeping the lower bound property in the resulting error assessment.

The continuous estimate $\boldsymbol{e}^{\star}$ can also be obtained using the recovery techniques proposed in section 3 or postprocessing the local solution of the residual type estimates based on Neumann local problems as described in Díez et al., 2003. Obviously, the quality of the resulting lower bound depends on how well $\boldsymbol{e}^{\star}$ approximates $\boldsymbol{e}$, in particular, for $\boldsymbol{e}^{\star}=\boldsymbol{e}, R\left(\boldsymbol{e}^{\star}\right)\left\|\boldsymbol{e}^{\star}\right\|^{-1}=\|\boldsymbol{e}\|$ and the estimate is therefore exact.

\section{CLOSURE}

The main techniques assessing the error associated with the FE discretization have been briefly reviewed. The tools available are progressively being incorporated in the FE commercial codes. From the end-user viewpoint, it is extremely important to have at hand one of these tools in order to evaluate the quality of the FE solution provided by the code. Ideally, the paradigm for the FE practitioner is to certify the quality of the solution for a given quantity of interest. The subsequent step is to adapt the mesh and design the optimal FE discretization, giving the desired answer with the prescribed accuracy at the minimum cost.

Adaptive strategies use the local error assessment to find the optimal element size in every zone of the domain. This can be done converting the local error into a desired element size using ad-hoc expressions derived from a priori estimates, see Díez and Calderón, 2007; Vidal et al., 2008. Then, a new mesh has to be build-up from scratch. A different approach consists in detecting the elements that need to be refined and refine the mesh keeping the structure of the previous one. If both upper and lower bounds of the quantity of interest are available, this can be done in terms of the local contribution to the bound gap. This local contribution is found to be positive in all the elements and, consequently, it can be used to assess which elements have to be refined. A complete description of the adaptive procedures is provided in a companion chapter of this Encyclopedia.

\section{REFERENCES}

Encyclopedia of Aerospace Engineering. Edited by Richard Blockley and Wei Shyy.

(c) 2010 John Wiley \& Sons, Ltd. 
Ainsworth, M. and Oden, J.T. A unified approach to a posteriori error estimation using element residual methods Numerische Mathematik 1993; 65(1):23-50.

Babuška, I. and Rheinboldt, W. C. Error estimates for adaptive finite element computations SIAM Journal on Numerical Analysis 1978; 15(4):736-754.

Bank, R. E. and Weiser, A. Some a posteriori error estimators for elliptic partial differential equations Mathematics of Computation 1985; 170(44):283-301.

Carstensen, C. and Funken, S.A. Fully reliable localized error control in the FEM SIAM Journal on Scientific Computing 1999/00; 4(21):1465-1484.

Chamoin, L. and Ladevèze, P. A non-intrusive method for the calculation of strict and efficient bounds of calculated outputs of interest in linear viscoelasticity problems Computer Methods in Applied Mechanics and Engineering 2008; 197(9-12):994-1014.

Díez, P. and Calderón, G. Remeshing criteria and proper error representations for goal oriented h-adaptivity Computer Methods in Applied Mechanics and Engineering 2007; 196(46):719-733.

Díez, P. and Egozcue, J.J. and Huerta, A. A posteriori error estimation for standard finite element analysis Computer Methods in Applied Mechanics and Engineering 1998; 163(14):141-157.

Díez, P. and Parés, N. and Huerta, A. Recovering lower bounds of the error by postprocessing implicit residual a posteriori error estimates International Journal for Numerical Methods in Engineering 2003; 10(56):1465-1488.

Fraeijs de Veubeke, B. Displacement and equilibrium models in the finite element method. In Zienkiewicz and Holister, editors, Stress Analysis. Wiley London, 1965. International Journal for Numerical Methods in Engineering, Classical Reprint Series 2001; 52:287-342.

Ladevèze, P. and Leguillon, D. Error estimate procedure in the finite element method and applications SIAM Journal on Numerical Analysis 1983; 20(3):485-509.

Ladevèze, P. and Maunder, E.A.W. A general method for recovering equilibrating element tractions Computer Methods in Applied Mechanics and Engineering 1996; 137(41):111151.

Ladevèze, P. and Pelle, J.-P. and Rougeot, Ph. Error estimation and mesh optimization for classical finite elements Engineering Computations. International Journal for Computeraided Engineering and Software 1991; 8(1):69-80.

Machiels, L. and Maday, Y. and Patera, A.T. A "flux-free" nodal Neumann subproblem approach to output bounds for partial differential equations Comptes Rendus de l'Académie des Sciences. Série I. Mathématique 2000; 3(330):249-254.

Morin, P. and Nochetto, R.H. and Siebert, K.G. Local problems on stars: a posteriori error estimators, convergence, and performance Mathematics of Computation 2003; 243(72):1067-1097.

Parés, N. and Bonet, J. and Huerta, A. and Peraire, J. The computation of bounds for linearfunctional outputs of weak solutions to the two-dimensional elasticity equations Computer Methods in Applied Mechanics and Engineering 2006; 195(4-6):406-429.

Encyclopedia of Aerospace Engineering. Edited by Richard Blockley and Wei Shyy.

(c) 2010 John Wiley \& Sons, Ltd. 
Parés, N. and Díez, P. and Huerta, A. Subdomain-based flux-free a posteriori error estimators Computer Methods in Applied Mechanics and Engineering 2006; 195(4-6):297-323.

Sauer-Budge, A. M. and Bonet, J. and Huerta, A. and Peraire, J. Computing bounds for linear functionals of exact weak solutions to Poisson's equation SIAM Journal on Numerical Analysis 2004; 42(4):1610-1630.

Vidal, Y. and Parés, N. and Díez, P. and Huerta, A. Bounds for quantities of interest and adaptivity in the element-free Galerkin method International Journal for Numerical Methods in Engineering 2008; 76(11):1782-1818.

Wiberg, N.E. and Li1, X.D. and Abdulwahab, F. Adaptive finite element procedures in elasticity and plasticity Engineering with Computers 1996; 12(2):120-141.

Xuan, Z. C. and Parés, N. and Peraire, J. Computing upper and lower bounds for the $J$ integral in two-dimensional linear elasticity Computer Methods in Applied Mechanics and Engineering 2006; 195(4-6):430-443.

Zienkiewicz, O.C. and Zhu, J.Z. A simple error estimator and adaptive procedure for practical engineering analysis International Journal for Numerical Methods in Engineering 1987; 24:337-357.

Zienkiewicz, O.C. and Zhu, J.Z. The superconvergent patch recovery (SPR) and adaptive finite element refinement Computer Methods in Applied Mechanics and Engineering 1992; 101:207-224.

\section{FURTHER READING}

Ainsworth, M. and Oden, J.T. A posteriori error estimation in finite element analysis. Pure and Applied Mathematics (New York). Wiley-Interscience [John Wiley \& Sons]: Chichester, 2000.

Babuška, I. and Strouboulis, T. The finite element method and its reliability. Numerical Mathematics and Scientific Computation. The Clarendon Press Oxford University Press: New York, 2001.

Bangerth, W. and Rannacher, R. Adaptive finite element methods for differential equations. Lectures in Mathematics ETH Zürich. Birkhäuser Verlag: Basel, 2003.

Ladevèze, P. and Pelle, J.P. Mastering calculations in linear and nonlinear mechanics. Mechanical Engineering Series. Translated from the 2001 French original by Theofanis Strouboulis. Springer-Verlag: New York, 2005.

Stein, E. (Editor) and Ramm, E. and Rank, E. and Rannacher, R. and Schweizerhof, K. and Stein, E. and Wendland, W. and Wittum, G. and Wriggers, P. and Wunderlich, W. Error-controlled Adaptive Finite Elements in Solid Mechanics. Wiley: Chichester, 2002.

Verfurth, R. A Review of a Posteriori Error Estimation and Adaptive Mesh-Refinement Techniques. Teubner Verlag and J. Wiley: Stuttgart, 1996. 